\title{
Cooperation of Syd-1 with Neurexin synchronizes pre- with postsynaptic assembly
}

\author{
David Owald ${ }^{1,2,7}$, Omid Khorramshahi ${ }^{1,2,7}$, Varun K Gupta ${ }^{1,2,7}$, Daniel Banovic ${ }^{3,4}$, Harald Depner ${ }^{1,2}$, \\ Wernher Fouquet $^{1,2}$, Carolin Wichmann ${ }^{1,2}$, Sara Mertel ${ }^{1,2}$, Stefan Eimer ${ }^{5}$, Eric Reynolds ${ }^{1,2}$, Matthew Holt ${ }^{1,6}$, \\ Hermann Aberle ${ }^{3,4}$ \& Stephan J Sigrist ${ }^{1,2}$
}

Synapse formation and maturation requires bidirectional communication across the synaptic cleft. The trans-synaptic NeurexinNeuroligin complex can bridge this cleft, and severe synapse assembly deficits are found in Drosophila melanogaster neuroligin (N/g1, dnlg1) and neurexin (Nrx-1, dnrx) mutants. We show that the presynaptic active zone protein Syd-1 interacts with Nrx-1 to control synapse formation at the Drosophila neuromuscular junction. Mutants in Syd-1 (RhoGAP100F, dsyd-1), Nrx-1 and NIg1 shared active zone cytomatrix defects, which were nonadditive. Syd-1 and Nrx-1 formed a complex in vivo, and Syd-1 was important for synaptic clustering and immobilization of Nrx-1. Consequently, postsynaptic clustering of NIg1 was affected in Syd-1 mutants, and in vivo glutamate receptor incorporation was changed in Syd-1, Nrx-1 and N/g1 mutants. Stabilization of nascent Syd-1-Liprin- $\alpha$ (DLiprin- $\alpha$ ) clusters, important to initialize active zone formation, was NIg1 dependent. Thus, cooperation between Syd-1 and Nrx-1-NIg1 seems to orchestrate early assembly processes between pre- and postsynaptic membranes, promoting avidity of newly forming synaptic scaffolds.

Understanding the process of synapse assembly in molecular and cell-biological detail is a prerequisite for understanding neural circuit development and activity-mediated remodeling, and thus is important for unraveling learning and memory processes (structural plasticity $)^{1-3}$. Functional chemical synapses are characterized by two apposed compartments that must be coestablished with high spatiotemporal precision: the presynaptic active zone, where regulated and rapid fusion of neurotransmitter-filled synaptic vesicles takes place, and the postsynaptic density (PSD), which embeds neurotransmitter receptors.

Glutamatergic neuromuscular junction (NMJ) terminals of Drosophila larvae grow to meet the requirements of the growing muscle fibers, a process in which new synapses are continuously added $^{4}$ (where a synapse is defined as a single active zone opposed by a single $\mathrm{PSD}^{1}$ ). In vivo imaging has shown that presynaptic Syd-1 and Liprin- $\alpha$ clusters initiate active zone formation ${ }^{5}$. On the postsynaptic side, initial PSD growth depends on incorporation of a glutamate receptor (GluR) containing the GluRIIA subunit. Later PSD maturation is marked by the incorporation of GluRIIB-containing receptor complexes ${ }^{6}$. Synapse assembly is concluded at presynaptic active zones by the incorporation of the active zone scaffold component Bruchpilot (BRP) ${ }^{5}$.

Coordinating synapse assembly requires signaling across the synaptic cleft, which separates pre- from postsynaptic membranes. Transsynaptic cell adhesion molecules are obvious candidates for coupling active zone and PSD assembly. In vitro, the Neurexin-Neuroligin (Nrx-Nlg) complex can mediate the trans-synaptic signaling required for synapse assembly 7,8 . How this signaling axis integrates with the additional assembly machinery, however, has remained largely unclear.

Here, we provide evidence of a dual role for Syd-1 in early assembly of NMJ synapses. It retains Liprin- $\alpha$ clusters at active zones and is important for clustering of presynaptic Nrx-1, likely through a direct and PDZ-domain-dependent interaction. Consequently, Syd-1 is also needed for clustering of postsynaptic Nlg1, which organizes postsynaptic assembly. Coincident action of Syd-1 with Nrx-1-Nlg1 appears to allow active zone scaffolds to pass through an initial, still fragile assembly phase. We suggest that binding between Syd-1 and Nrx-1Nlg1 is a means to coordinate pre- with postsynaptic assembly. Our study shows an example of how coincident action of a presynaptic active zone scaffold protein and a trans-synaptic cell adhesion protein module can spatiotemporally orchestrate synapse assembly.

\section{RESULTS}

Initially described in cell culture systems (for a review, see ref. 9), interaction between mammalian presynaptic Nrx proteins and postsynaptic Nlg molecules was proposed to be important for proper synapse assembly. However, genetic ablation of three $N \lg (N \operatorname{lgn})$ genes in mice ${ }^{10}$ does not result in a substantial structural phenotype, potentially reflecting a strong capacity for compensatory processes in vivo.

Nonetheless, severe deficits in the in vivo assembly of Drosophila NMJ synapses ${ }^{11-13}$ were reported for mutants of Nrx-1 and Nlg1 (for domain organization, see Fig. 1a). These findings provided an opportunity for

${ }^{1}$ Freie Universität Berlin, Institute for Biology/Genetics, Berlin, Germany. ${ }^{2}$ NeuroCure, Charité, Berlin, Germany. ${ }^{3}$ University of Münster, Institute for Neurobiology, Münster, Germany. ${ }^{4}$ Heinrich Heine University Düsseldorf, Functional Cell Morphology Lab, Düsseldorf, Germany. ${ }^{5}$ European Neuroscience Institute, Göttingen, Germany. ${ }^{6}$ VIB Center for the Biology of Disease, Leuven, Belgium. ${ }^{7}$ These authors contributed equally to this work. Correspondence should be addressed to S.J.S. (stephan.sigrist@fu-berlin.de). 
Figure 1 Double mutants analysis of $\mathrm{Nrx}-1$, NIg1 and Syd-1. (a) Domain structures. Nrx-1 includes extracellular laminin G (LAM G) and epidermal growth factor (EGF)-3 domains. The short intracellular domain has a PDZ-binding motif. The extracellular part of NIg1 comprises an acetylcholinesterase-like (AChE) domain, while the cytoplasmic part has a PDZ-binding motif. The D356R point mutation $\left({ }^{*}\right)$ in the AChE-like domain interferes with $\mathrm{Nrx}-1$ binding. The cytoplasmic part is deleted in Nlg1 $\Delta$ Cyto

comprises a PDZ domain, a C2 domain and a putative RhoGAP domain. PDZ* indicates the Syd-1 R165A L170A point mutations

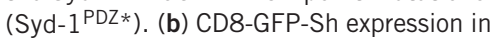
NMJs 1 and 9. Scale bar, $20 \mu \mathrm{m}$. (c) Quantification of bouton numbers on NMJs 1 and 9 relative to muscle area. Control: $5.185 \pm 0.171$ boutons per $10^{4} \mu \mathrm{m}^{2}, n=20$; UAS-Nlg1 $\Delta$ Cyto: $0.850 \pm$ $0.205, n=20$; UAS-NIg1 $\Delta$ Cyto ${ }^{D 356 R}: 3.84 \pm$ $0.221, n=20 ; N r x-1: 3.225 \pm 0.178, n=20$; Nrx-1,UAS-NIg1 1 Cyto: $3.205 \pm 0.209, n=20$; Liprin- $\alpha$ : $3.935 \pm 0.164, n=20$; Liprin- $\alpha$, UAS-NIg1 $\Delta$ Cyto: $1.374 \pm 0.269, n=20$; Syd-1: $3.953 \pm 0.199, n=20$; Syd-1, UAS-Nlg1 $\Delta$ Cyto: $3.537 \pm 0.21, n=20 ; N / g 1: 3.105 \pm 0.170$, $n=19 ;$ Syd-1,Nrx-1: $3.505 \pm 0.230, n=20$; Syd-1, Nlg1: $3.200 \pm 0.246, n=18$. All single and double mutant data were analyzed using the Kruskal-Wallis test with Dunn's multiple comparison test (test statistic $K=48.77$ ).

Each single mutant was compared to UAS-NIg1 $\Delta$ Cyto expressed in the mutant background using the Mann-Whitney U-test: Nrx-1 versus $N r x-1$, UAS-NIg1 $\Delta$ Cyto $P=0.59, U=179.5$; Liprin- $\alpha$ versus Liprin- $\alpha$,UAS-NIg1 $\Delta$ Cyto $P<0.001, U=15.0$; Syd-1 versus Syd-1,UAS-NIg1 $\Delta$ Cyto $P=0.22$, $U=138$.0. Control, UAS-NIg1 $\triangle$ Cyto and UAS-NIg1 $\triangle$ Cyto ${ }^{\mathrm{D} 356 \mathrm{R}}$ were analyzed using the Kruskal-Wallis test with Dunn's multiple comparison test $(K=44.73) .{ }^{*} P<0.05 ;{ }^{* *} P<0.01 ;{ }^{* *} P<0.001 ; \mathrm{NS}$, not significant $(P>0.05)$; all values are mean \pm s.e.m. a
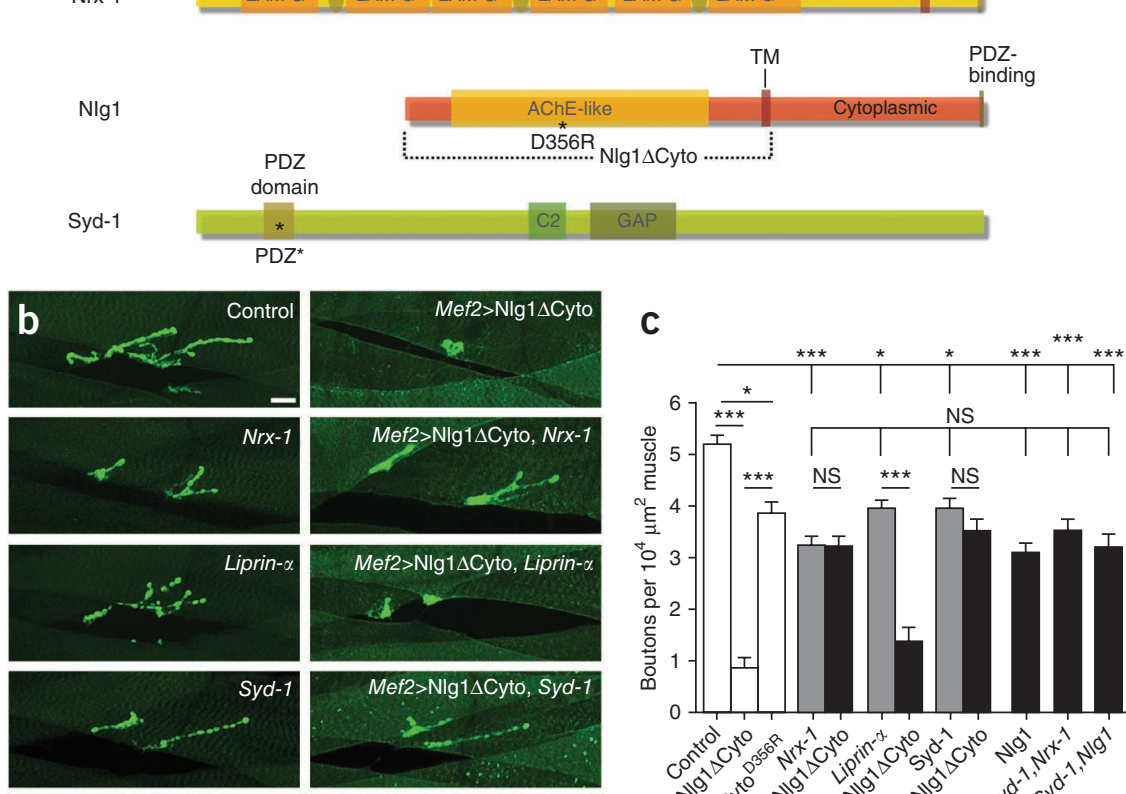

a
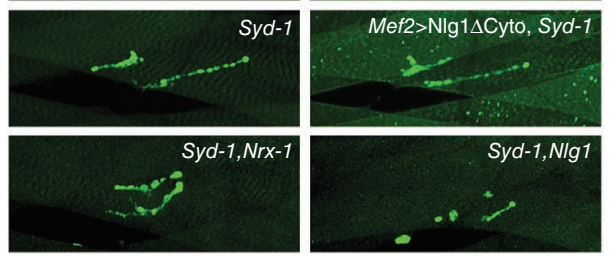

an efficient study of how this trans-synaptic signaling complex integrates into synapse assembly in a genetically tractable in vivo model.

We started by searching for additional factors involved in the Nlg1-instructed role of Nrx-1. Previously, we found that muscle (Mef2-Gal4-driven) expression of GFP-tagged Nlg1 lacking its $\mathrm{C}$ terminus (UAS-Nlg1 $\Delta$ Cyto $^{\mathrm{GFP}}$ (ref. 12), Fig. 1a) results in marked under-growth of NMJs (Fig. 1b,c). Therefore, postsynaptic Nlg1 $\Delta$ Cyto has a dominant negative effect on the addition of presynaptic boutons (and thus active zones) during terminal expansion. Although the mechanism underlying this phenotype is still elusive, we noted that introducing a point mutation interfering with Neurexin binding $^{14}$ (UAS-Nlg1 1 Cyto $^{\text {D356R }}$, Fig. 1a) largely suppressed the dominant negative effect of Nlg1 $\Delta$ Cyto (Fig. 1c). Likewise, expression of Nlg1 $\Delta$ Cyto in the Nrx-1 mutant background did not lead to the severe under-growth Nlg1 $\Delta$ Cyto caused in the control background. Instead, NMJ structures and bouton numbers were indistinguishable from those of $N r x-1$ mutants (Fig. 1b,c). Thus, as the Nlg1 $1 \Delta$ Cyto phenotype depends on Nrx-1, testing for suppression of Nlg1 $\Delta$ Cyto might be a way to identify additional presynaptic factors implicated in Nrx-1 signaling.

In both Drosophila and Caenorhabditis elegans, presynaptic Syd-1 (for domain organization, see Fig. 1a) and Liprin- $\alpha$ (Syd-2) promote presynaptic assembly ${ }^{5,15-18}$. When we expressed Nlg1 $\Delta$ Cyto in a Liprin- $\alpha$ mutant background, NMJ terminals still showed severe under-growth compared to those in the Liprin- $\alpha$ mutant background alone (Fig. 1b,c). In contrast, the Syd-1 mutant background fully suppressed the Nlg1 $1 \Delta$ Cyto-induced NMJ under-growth (Fig. 1b,c), implying that Syd-1 and Nlg1 may operate in the same pathway.

\section{Similar synaptic phenotypes of Syd-1, Nrx-1 and N/g1}

We previously showed that $N r x-1$ and $N \lg 1$ behave nonadditively: the reduced NMJ bouton numbers in Nlg1 mutants are not further diminished in a $N \lg 1, N r x-1$ double mutant ${ }^{12}$. To test whether Syd-1 genetically interacts with $N r x-1$ or $N l g 1$, we constructed the respective double mutants. NMJ size is reduced in Nlg1, Nrx-1 and Syd-1 single mutants, as well as in Liprin- $\alpha$ single mutants ${ }^{5,11,12,18}$. As expected, all these single mutants showed significantly reduced bouton numbers as compared to controls (Fig. 1b,c). Both Syd-1,Nrx-1 and Syd-1,Nlg1 double mutants reached third instar larval stage, allowing bouton number analysis at mature NMJs (whereas Syd-1,Liprin- $\alpha$ double mutants die as embryos ${ }^{5}$ ). Combination of $N r x-1$ or $N \lg 1$ with the Syd-1 mutant did not reduce bouton numbers further than those of the individual single mutants (Fig. 1b,c).

Apart from reduced NMJ size, all three mutants (Nlg1, Nrx-1 and Syd-1) show defects in the organization of the remaining active zones $^{5,11,12}$. We compared their active zone organization by using stimulated emission depletion $\left(\right.$ STED $^{19}$ ) microscopy at $\sim 80 \mathrm{~nm}$ resolution (instead of the $\sim 250 \mathrm{~nm}$ of a standard confocal microscope) and electron microscopy. In electron micrographs of controls, active zone dense bodies (T-bars) were properly shaped (Fig. 2a). In contrast, an aberrant, star-shaped T-bar morphology was regularly observed at the remaining active zones of Syd-1 (compare ref. 5), Nrx-1 and Nlg1 mutants, but never at control active zones (Fig. 2a-f).

The protein BRP is a molecular building block of the electrondense T-bar ${ }^{5,20}$. At control NMJs, diffraction-limited BRP spots mark individual active zones ${ }^{21}$ that appear as ring-shaped structures when imaged at higher resolutions with STED ${ }^{20,22}$ (Fig. 2g,h). We stained 

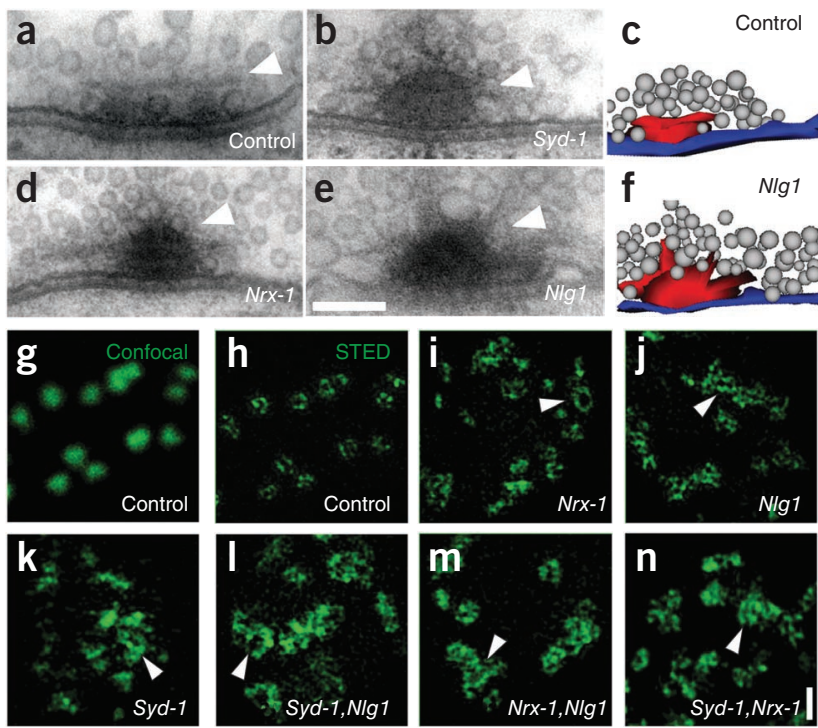

0

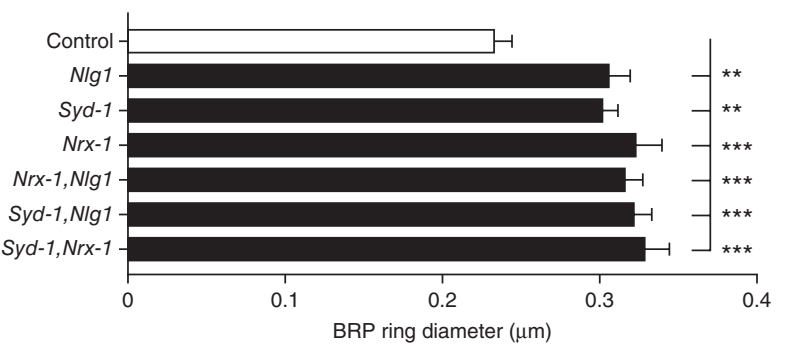

Nrx-1 (Fig. 2i) and Nlg1 (Fig. 2j) mutants for BRP and imaged active zones using STED. BRP rings were frequently interconnected and over-grown (Fig. 2i,j). We had previously observed this phenotype in STED images of $S y d-1$ mutants ${ }^{5}$ (Fig. 2k). In fact, BRP ring diameters were increased in all Syd-1 (compare ref. 5), Nrx-1 and Nlg1 single mutants, which were statistically indistinguishable from each other and from the double mutants (Fig. 2i-o).

\section{Syd-1 is needed to cluster Nrx-1 and NIg1 at NMJ synapses}

Endogenous Nrx-1 forms distinct clusters at the presynaptic terminal (Fig. 3a) ${ }^{11,12}$. We performed immunostaining to investigate whether Syd-1 could influence the distribution of Nrx-1. As with Nlg1 mutants (Fig. 3a,b), Nrx-1 intensity was significantly reduced in Syd-1 mutants (Fig. 3a,b).

Nlg1 normally clusters opposite presynaptic Nrx-1. These clusters are no longer detectable in Nrx-1 mutants (compare ref. 12). Consistent with Syd-1 organizing Nrx-1 clustering, Nlg1 clusters also dropped below the detection limit in Syd-1 mutants (Fig. 3c,d).

Both Syd-1 and Nrx-1 mutant terminals show reduced glutamate release ${ }^{5,11}$. Thus, lack of Nlg1 clustering could be a mere consequence of reduced synaptic activity. To test this, we quantified Nlg1 intensity (as a measure of Nlg1 clustering) in mutants for presynaptic $b r p$ $\left(b r p^{1.3}\right)^{20}$. The $b r p$ mutant larvae show a stronger decrease in presynaptic neurotransmitter release than any of the mutants discussed above ${ }^{5,11,20,22,23}$. Nlg1 clusters, however, were not reduced in $b r p$ mutants (Fig. 3c,d), indicating that the observed reduction of Nlg1 in Syd-1 and Nrx-1 mutants was neither due to reduced transmission nor a reflection of a general active zone assembly deficit (which is very pronounced in $b r p$ mutants $\mathrm{s}^{22}$ ). Instead, Syd-1 seems to be specifically involved in clustering postsynaptic Nlg1 adjacent to synapses.

The finding that the cytoplasmic scaffold protein Syd-1 is needed for both Nrx-1 and Nlg1 clustering is consistent with Nrx-1 directly
Figure 2 Comparison of active zone ultrastructure for Nrx-1, N/g1 and Syd-1 mutants. (a-f) Electron microscopy of presynaptic electron-dense projections (T-bars). (a) A normal presynaptic electron-dense projection (T-bar) of a control bouton. (c) Serial reconstruction of the T-bar shown in a. T-bars of Syd-1 (b), Nrx-1 (d), and Nlg1 (e) mutant active zones often show star-like shapes. Arrowheads indicate T-bars. (f) Serial reconstruction of the N/g1 mutant T-bar shown in e. Scale bar, $100 \mathrm{~nm}$. (g,h) Examples of confocal (g) and STED (h) images of BRP spots at a control NMJ (muscle 4). (i-n) BRP rings are irregular and interconnected (arrowheads) in Nrx-1 (i), NIgl (j) and Syd-1 (k) single mutants and Syd-1,N/g1 (I), Nrx-1,Nlg1 (m) and Syd-1,Nrx-1 (n) double mutants. STED images were deconvolved with a Lorentz point spread function of $80 \mathrm{~nm}$ using a Wiener filter $(0.05$ regulation parameter). Scale bar, $500 \mathrm{~nm}$. (o) Quantification of BRP ring diameter. Control: $0.233 \pm 0.012 \mu \mathrm{m}, n=34$; N/g1: $0.305 \pm 0.015$, $n=43 ;$ Syd-1: $0.301 \pm 0.011, n=47 ;$ Nrx-1: $0.322 \pm 0.013, n=54$; Nrx-1,N/g1: $0.315 \pm 0.013, n=52 ;$ Syd-1,N/g1: $0.321 \pm 0.013, n=46$; Syd-1,Nrx-1: $0.328 \pm 0.017, n=43$. Kruskal-Wallis test with Dunn's multiple comparison test $(K=29.2)$. ${ }^{*} P<0.05 ;{ }^{* *} P<0.01 ;{ }^{* * *} P<0.001$; NS, not significant; all values are mean \pm s.e.m.

organizing the synaptic clustering of Nlg1. We also found that musclespecific Nlg1 expression markedly increased presynaptic Nrx-1 clustering in the control background (Supplementary Fig. 1a) and that there was a trend for motor neuron-driven UAS-Nrx-1 to increase postsynaptic Nlg1 (Fig. 3e,f). We reasoned that if $\mathrm{Nrx}-1$ availability was rate limiting for Nlg1 clustering in Syd-1 mutants, overexpression of Nrx-1 in Syd-1 mutants might allow restoration of postsynaptic $\mathrm{Nlg} 1$ clustering. Indeed, $\mathrm{Nlg} 1$ clustering was partially restored when we overexpressed Nrx-1 in the Syd-1 mutant background (Fig. 3e,f). Of note, immunostainings for endogenous Syd- 1 showed that the protein was still detectable at both Nrx-1 and Nlg1 mutant NMJs (Supplementary Fig. 2). Taken together, these results show that Syd-1 is needed to effectively cluster Nrx-1, which likely affects the clustering of postsynaptic Nlg1.

\section{Syd-1 and Nrx-1 form an in vivo complex}

These findings motivated us to analyze whether Syd-1 and Nrx-1 might be part of a common complex. We performed immunoprecipitations from an active zone protein-enriched preparation, derived from Drosophila head extracts, using antibodies recognizing Nrx-1 (ref. 24). There was robust coimmunoprecipitation of Syd-1 with Nrx-1, whereas specificity controls were negative (Fig. 4a).

We next sought the molecular link joining Syd-1 and Nrx-1. Syd-1 comprises a PDZ domain, and Nrx-1 has a PDZ-binding motif (Fig. 1a). A large-scale C. elegans yeast two-hybrid screen recently identified a direct interaction of the Syd-1 PDZ domain with C. elegans Nrx in vitro ${ }^{25}$. We found the same interaction using the corresponding stretches of the fly proteins (Supplementary Fig. 3a). We introduced two point mutations (Syd-1 R165A L170A (Syd-1 ${ }^{\text {PDZ*}}$ ); compare ref. 26) into the Syd-1 cDNA to destroy the PDZ domain's ligand-binding capability. When tested in a yeast two-hybrid assay, binding of the Nrx-1 C terminal to the mutated Syd-1 PDZ domain was greatly reduced as compared to its binding to the unmutated Syd-1 PDZ domain (Supplementary Fig. 3a).

Expressing $\mathrm{Nrx}-1^{\mathrm{GFP}}$ (Fig. 4b) and Syd-1 tagged with the fluorescent protein mStrawberry ( ${ }^{\mathrm{mStraw}}$ Syd-1) (Fig. 4c) in larval salivary gland cells, we tested this interaction in vivo. As expected, when expressed by itself, the transmembrane protein Nrx-1 labeled the plasma membrane (Fig. 4b). In contrast, Syd-1 or Syd-1 ${ }^{\text {PDZ* }}$ expressed on its own had an intracellular distribution (Fig. 4c,d). When expressed with Nrx-1, however, Syd-1 was clearly recruited to the plasma membrane (Fig. 4e), whereas Syd-1 ${ }^{\text {PDZ*}}$ was not (Fig. 4f). In the reverse experiment, Nrx-1 lacking its PDZ-binding motif (UAS-Nrx-1 ${ }^{\left.\mathrm{PDB}^{*}, \text { no-tag; ref. } 24\right) \text { failed to }}$ 
Figure 3 Reduced endogenous Nrx-1 and NIg1 clusters at Syd-1 mutant NMJs. (a) Muscle 4 boutons of control, N/g1, Syd-1 and Nrx-1 larvae immunostained for Nrx-1 and BRP. (b) Quantification of Nrx-1 signal intensities from NMJs on muscle 4, normalized to control. Residual background (mean intensity of $\mathrm{Nrx}-1$ mutants) was subtracted for all genotypes and data sets were then normalized to control. Control: $100 \pm 12.40, n=21 ;$ Syd-1: $29.44 \pm 6.58, n=19 ; \mathrm{N} / g 1: 34.46 \pm 5.06$, $n=18$; Nrx-1: $0.00 \pm 1.43, n=18$; control versus Syd-1 $P<0.001$; control versus NIg1 $P<0.01$; control versus Nrx-1 $P<0.001$; Syd-1 versus NIg1 $P>0.05$; Kruskal-Wallis test with Dunn's multiple comparison test $(K=52.6)$. (c) Muscle 4 boutons of control, N/g1, Syd-1, and brp larvae immunostained for NIg1 and BRP. (d) Quantification of NIg1 signal intensity from NMJs on muscle 4 . Residual background (mean intensity of NIg1 mutants) was subtracted for all genotypes (hence the negative values for Syd-1 mutants) and data sets were then normalized to control. Control: $100 \pm 14.61, n=14$; Syd-1: $-20.8 \pm$ 7.34, $n=9$; Nlg1: $0.0 \pm 19.36, n=8$; brp: $90.1 \pm 38.95, n=7$; control versus Syd-1 $P<0.001$; control versus NIg1 $P<0.01$; control versus brp $P>0.05$; Kruskal-Wallis test with Dunn's multiple comparison test $(K=22.4)$. (e) Muscle 4 boutons of control larva and of control and Syd-1 larvae with UAS-Nrx-1 GFP driven in motor neurons. (f) Quantification of NIg1 signal intensities normalized to control. Control: $100 \pm 10.40, n=12$; Nrx-1 GFP: $195.0 \pm 45.59, n=13$; Syd-1: $8.505 \pm 1.390, n=13$; Nrx-1 ${ }^{\text {GFP }}$, Syd-1: $36.43 \pm 4.202 ; n=12$; control versus Nrx-1GFP $P>0.05$; Mann-Whitney U-test $(U=55.0)$; Syd- 1 versus Nrx-1 GFP, Syd-1 $P<0.001$; Mann-Whitney $U$-test $(U=0.0)$. NS, not significant; ${ }^{* *} P<0.01,{ }^{* * *} P<0.001$; all values are mean \pm s.e.m. Scale bars, $2 \mu \mathrm{m}$.

recruit ${ }^{\text {mStraw }}$ Syd-1, whereas the control, UAS-Nrx-1 ${ }^{\text {no-tag }}$ (ref. 24), effectively recruited ${ }^{\mathrm{mStraw}}$ Syd- 1 to the plasma membrane (Supplementary Fig. 3b). Thus, Nrx-1 and Syd-1 can be found in a common complex in vivo, with their binding apparently mediated through a direct PDZdomain interaction.

\section{Syd-1 recruits Nrx-1 to active zones}

We next performed in vivo imaging experiments ${ }^{6}$ (Fig. 5a) at NMJs of developing intact larvae. As expected ${ }^{5}$, motor neuron-driven (ok6-Gal4) UAS-mStrawSyd-1 invariably marked active zones. UAS-Nrx-1 ${ }^{\text {GFP }}$, when expressed alone, however, distributed more diffusely over the presynaptic terminal membrane (Fig. 5b) and was only slightly enriched close to the active zones marked by BRP (data not shown). When we expressed both proteins under these conditions, Nrx-1 GFP became densely enriched in the Syd-1-positive active zones (compare Fig. 5b and Fig. 5c). Thus, overexpressed Syd-1 can direct exogenous Nrx-1 to active zones. Next we expressed UAS-Syd-1 ${ }^{\text {PDZ* }}$. UAS-Syd-1 ${ }^{\text {PDZ* }}$ still localized to active zones to a fair extent (Fig. 5d), but coexpressed Nrx-1 appeared diffuse (Fig. 5d), with a distribution indistinguishable from that in the absence of Syd-1 expression (Fig. 5b). Thus,

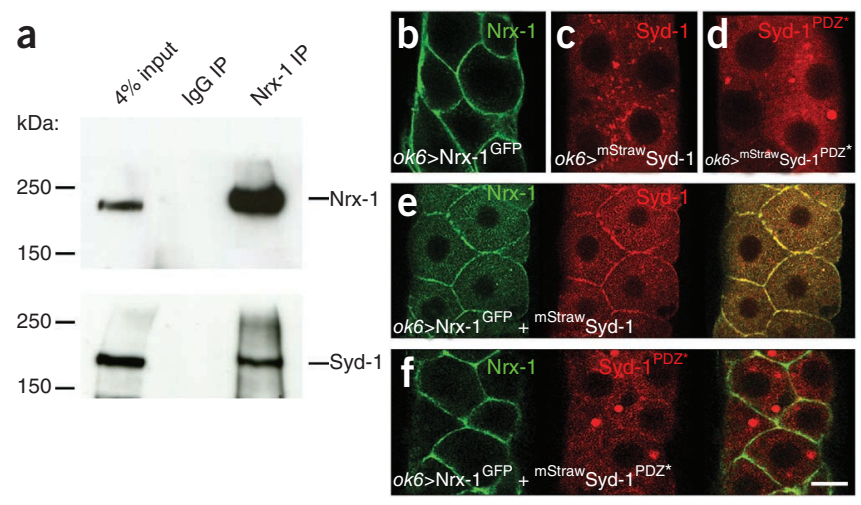

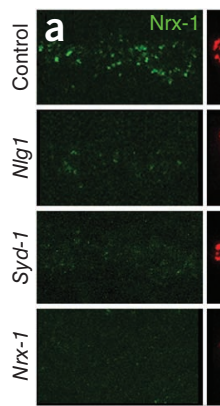
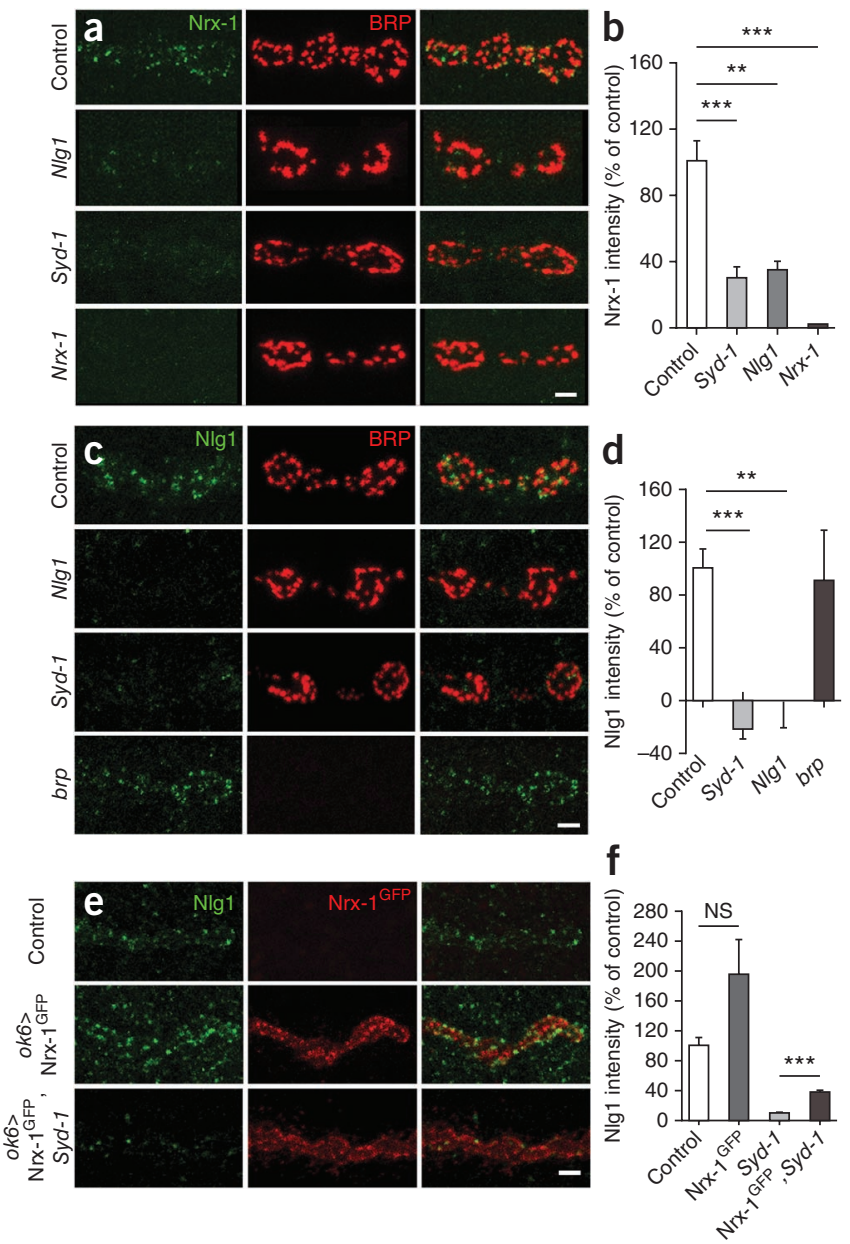

Syd- $1^{\text {PDZ*}}$ failed to recruit Nrx-1 to active zones. Liprin- $\alpha$ overexpression was not sufficient to trigger active-zone enrichment of $\mathrm{Nrx}-1$ GFP (Supplementary Fig. 4a).

We also noted that endogenous postsynaptic Nlg1 clustering was reduced at terminals that presynaptically overexpressed UASSyd-1 ${ }^{\text {PDZ* }}$ (Supplementary Fig. 1b). Syd-1 ${ }^{\mathrm{PDZ} *}$ likely competes with endogenous Syd-1 at active zones, impairing Nrx-1-Syd-1 interactions and, thereby, Nlg1 clustering.

We next used STED to investigate Nrx-1 localization relative to the active zone core marker BRP. At $\sim 80 \mathrm{~nm}$ resolution, Syd-1 puncta surround the BRP core ${ }^{5}$. A good fraction of Nrx-1 ${ }^{\text {GFP }}$ puncta also surrounded the BRP core when expressed with Syd-1. This active zone-associated distribution (Fig. 5e) was no longer apparent when we expressed Nrx-1 with Syd-1 ${ }^{\text {PDZ }}{ }^{\star}$ (Fig. 5f); in this case, Nrx-1 puncta not associated with the active zone dominated instead.

Figure 4 Biochemical and cell biological analysis of Syd-1-Nrx-1 complex. (a) Immunoblot of Nrx-1 immunoprecipitate (IP) from a Drosophila active zone protein-enriched preparation. Syd-1 coprecipitates with $\mathrm{Nrx}-1$ but is not detected in the random mouse immunoglobulin G (IgG) control. For full blots, see Supplementary Figure 10. (b) UASNrx-1 GFP localizes to the cell membrane of salivary gland cells, whereas (c,d) either UAS-mStrawSyd-1 or UAS-mStrawSyd-1 ${ }^{\text {PDZ* }}$ expressed alone distributes diffusely throughout the cytoplasm. (e) Coexpression of UAS-mStrawSyd-1 and UAS-Nrx-1 GFP directs UAS-mStrawSyd- 1 to the plasma membrane. (f) In contrast, localization of UAS-mStraw Syd-1 ${ }^{\text {PDZ* }}$ expressed with UAS-Nrx-1 ${ }^{\text {GFP }}$ remains diffuse. Scale bar, $25 \mu \mathrm{m}$. 
a

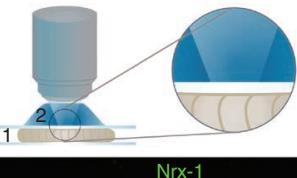

C

d

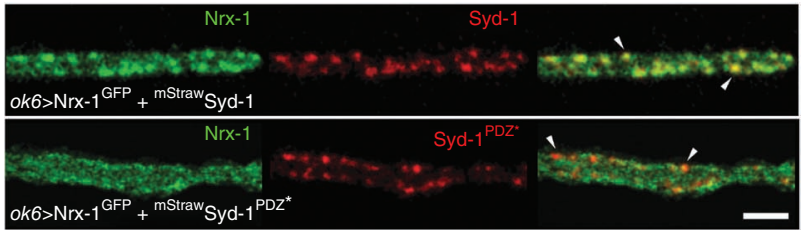

Figure 5 Syd- 1 influences localization and FRAP of Nrx-1 at NMJ. (a) The in vivo imaging procedure at Drosophila larval NMJs. Identified synapses are reimaged at defined time intervals in an intact larva. (1) Larva between cover slips. (2) Oil immersion objective. (b) Junctional localization of UAS-Nrx-1 GFP driven in motor neurons (muscle 26/27). Scale bar, $2 \mu \mathrm{m}$. (c) Junctional localization of UAS-Nrx-1 GFP expressed with UAS-mStrawSyd-1. Arrowheads indicate clusters of Syd-1 and Nrx-1 near the active zone. (d) Junctional localization of UAS-Nrx-1 ${ }^{\text {GFP }}$ expressed with UAS-mStrawSyd-1 ${ }^{\text {PDZ* }}$. Arrowheads indicate missing overlap of Syd-1 and Nrx-1 near the active zone. Scale bar (applies to $\mathbf{c}, \mathbf{d}$ ), $2 \mu \mathrm{m}$. (e,f) Single confocal or STED sections of boutons triple-labeled for UAS-Nrx-1 GFP and endogenous BRP with, respectively, mStrawSyd-1 (e) or mStrawSyd-1 PDZ* (f). STED images of UAS-Nrx-1 GFP reveal the subsynaptic distribution of Nrx-1. When expressed with Syd-1, Nrx-1 clusters localize adjacent to active zones labeled by BRP. When expressed with Syd-1 ${ }^{\mathrm{PDZ} *}$, the distribution of UAS-Nrx-1 appears randomized and not enriched near active zones. Scale bar, $500 \mathrm{~nm}$. (g) FRAP of UAS-Nrx-1 GFP driven in motor neurons of Syd-1 mutant, Syd-1,UAS-Syd-1 rescue (Syd-1 R) and Syd-1,UAS-Syd-1 PDZ $^{*}$ (Syd-1 PDZ $^{*}$ ) larvae. The middle (photobleached) row was taken $\sim 1-2$ min after the top. Scale bar, $2 \mu \mathrm{m}$. (h) Quantification of the Nrx-1 recovery signal. Nrx-1 FRAP was normalized to the recovery of Nrx-1 in Syd-1 mutants. Syd-1: $1 \pm 0.087, n=15$; Syd-1,UAS-Syd-1 (Syd-1 R): $0.320 \pm 0.070, n=12$; Syd-1,UAS-Syd-1PDZ* (Syd-1 PDZ*): 1.171 $\pm 0.063, n=13$; Syd-1 versus Syd-1 R, $P<0.001$; Syd-1 R versus Syd-1 PDZ*, $P<0.001$; Kruskal-Wallis test with Dunn's multiple comparison test $(K=23.5),{ }^{* *} P<0.001$; all values are mean \pm s.e.m.

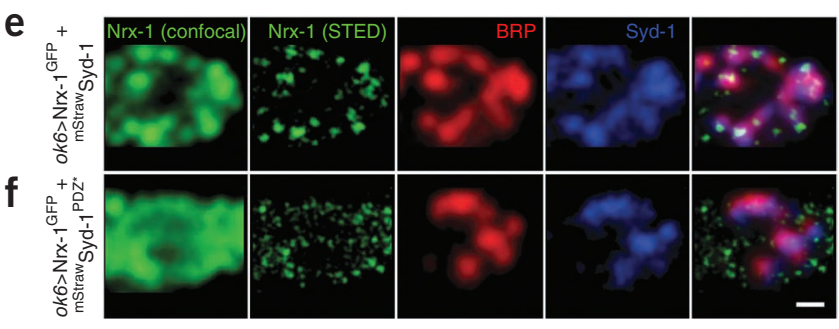

g
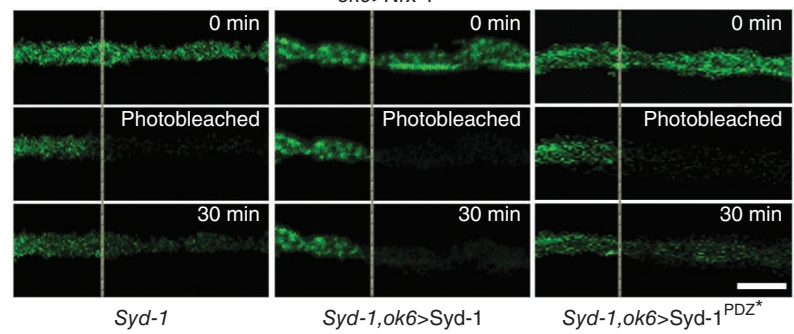

h

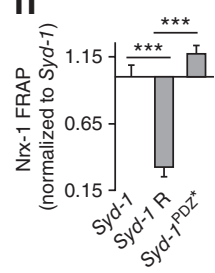

\section{Syd-1 retains Liprin- $\alpha$ and Nrx-1 at synapses}

It is conceivable that the synapse assembly process may demand continuous trafficking and remodeling. Notably, in the hierarchy of active zone assembly, genetic analysis in C. elegans has placed Syd-1 upstream of Syd-2 (Liprin- $\alpha$ ), a protein linked to long-range transport of vesicular axonal cargo $^{27,28}$.

We previously found that Liprin- $\alpha$ localization was severely disturbed in Syd-1 mutants and proposed that Syd-1 might anchor clusters of Liprin- $\alpha^{5}$. To address this, we imaged Liprin- $\alpha$ clusters of control and Syd-1 NMJs in vivo in 30-min increments (Supplementary Fig. 5a) and scored mobile spots (comparing images taken at 0 and
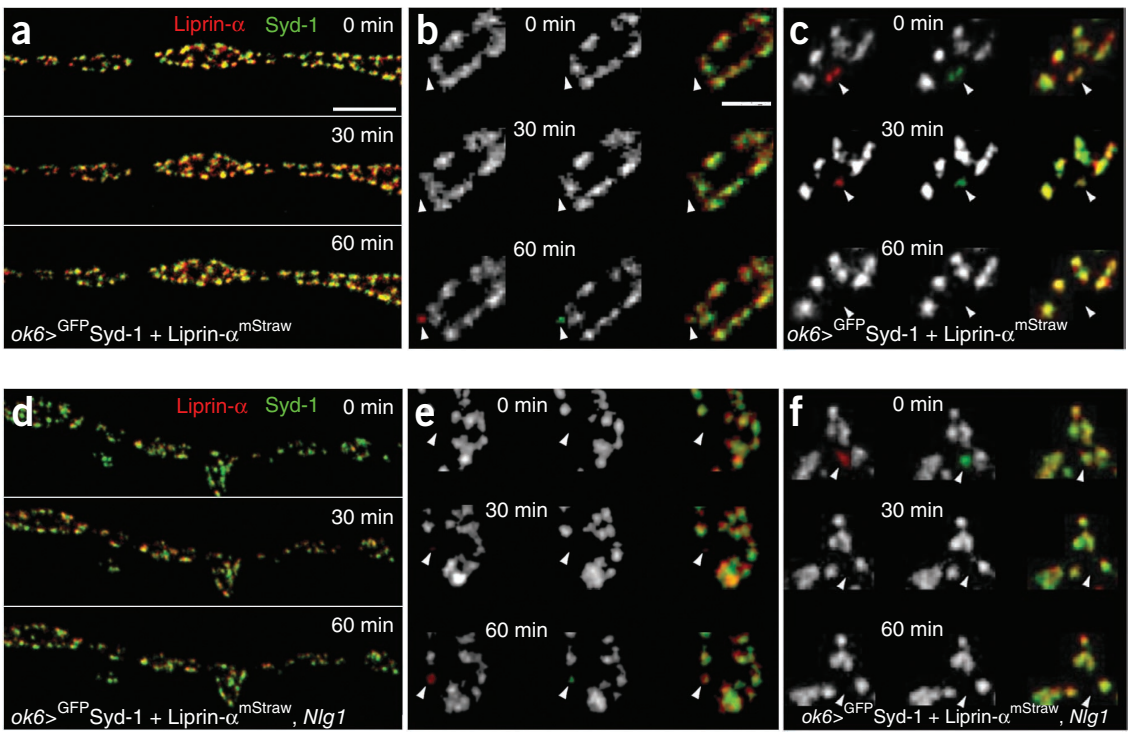

$60 \mathrm{~min}$; see Online Methods). Numbers of mobile Liprin- $\alpha$ spots were elevated in the Syd-1 mutant background (Supplementary Fig. 5b). Notably, this interaction was not reciprocal, as Syd-1 mobility appeared unchanged in Liprin- $\alpha$ mutants (data not shown).

Does Syd-1 retain Nrx-1 in a similar manner? Owing to the diffuse nature of overexpressed Nrx-1 ${ }^{\mathrm{GFP}}$, tracking of individual clusters was problematic here. Instead, we performed fluorescence recovery after photobleaching (FRAP) experiments at Nrx-1 ${ }^{\mathrm{GFP}}$-expressing synaptic terminals and measured recovery of the diffuse GFP signal (Fig. 5g,h). We compared NMJs expressing Nrx-1 ${ }^{\mathrm{GFP}}$ in Syd-1 mutants; in a rescue experiment, with Syd-1 reexpressed in the Syd-1 mutant background; and with Syd-1 ${ }^{\text {PDZ* }}$ re-expressed in the Syd-1 mutant background (Fig. 5g,h). The Syd-1 mutants showed higher Nrx-1 ${ }^{\text {GFP }}$ mobility than rescue controls. Notably, UAS-Syd-1 ${ }^{\mathrm{PDZ}}{ }^{\star}$ expression in the Syd-1 mutant background also

Figure 6 In vivo imaging of Liprin- $\alpha-$ Syd-1 cluster dynamics at N/g1 mutant NMJs. (a) In vivo-imaged NMJ boutons from a UASGFPSyd-1 and UAS-Liprin- $\alpha^{\text {mStraw-expressing }}$ larva (muscle 26/27). Junctions were reimaged after 30 and 60 min. (b) A new Liprin- $\alpha$ (red, arrowheads)- and Syd-1 (green, arrowheads)positive site emerging at $60 \mathrm{~min}$ (as expected ${ }^{5}$ ). (c) A nascent site disassembling again; arrowheads and colors in c,e,f as in b. (d) In vivo-imaged NMJ boutons from a UAS-GFPSyd-1- and UASLiprin- $\alpha^{\text {mStraw }}$-expressing Nlg1 mutant. (e,f) New Liprin- $\alpha$ - and Syd-1-positive sites assembling (e), disassembling (f) and moving. Scale bar in a,d, $5 \mu \mathrm{m}$. Scale bar in $\mathbf{b}, \mathbf{c}, \mathbf{e}, \mathbf{f}, 1 \mu \mathrm{m}$. 
Figure 7 Analysis of in vivo glutamate receptor incorporation in N/g1, Nrx-1 and Syd-1 mutants. (a) Time-lapse ( $24 \mathrm{~h}$ ) of growing NMJs at muscles 26/27 for the determination of GluR content of newly forming PSDs. At control NMJs, most of the young PSDs (arrowheads) are rich in GluRIIA, with a few exceptions (asterisks). Young PSDs in Nrx-1, Syd-1 and N/g1 mutants (genotypes indicated in black) show an inverse trend in GluRIIA-GluRIIB composition (arrowheads). (b) The GluRIIB/IIA composition $^{6}$ in newly formed PSDs is significantly shifted toward GluRIIB, in comparison to control PSDs, for all Nrx-1, Syd-1 and N/g1 mutants. Control: $-0.283 \pm 0.038, n=23 ; N r x-1:-0.019$ $\pm 0.038, n=20 ;$ Syd-1: $0.064 \pm 0.035, n=16$; NIg1: $0.114 \pm 0.026, n=30$; control versus Nrx-1 $P<0.01$; control versus Syd-1 $P<0.001$; control versus N/g1 $P<0.001$; Kruskal-Wallis test with Dunn's multiple comparison test $(K=39.1)$; ${ }^{* *} P<0.01,{ }^{* * *} P<0.001$, all values are mean \pm s.e.m. (c) Subsynaptic localization of GluRIIAand GluRIIB-type glutamate receptor subunits at individual PSDs on muscle 4 of larvae (fixed preparation). GluRIIB localizes to the edge of a GluRIIA-rich core in most control PSDs. For Nrx-1, Syd-1 and N/g1 mutant NMJs, examples are shown in which this distinct localization is reversed. Scale bars, $5 \mu \mathrm{m}$.

resulted in a very high recovery of bleached Nrx- $1^{\text {GFP }}$ signal. In contrast, Nrx-1 ${ }^{\text {GFP }}$ recovery appeared unchanged in both Liprin- $\alpha$ mutants and a Liprin- $\alpha$-overexpressing background (Supplementary Fig. 4b). These findings suggest that Syd-1, but not Liprin- $\alpha$, is able to retain Nrx-1.

During these live imaging experiments, we observed that Nrx-1 GFP levels were reduced in Syd-1 mutants, as compared to those in the control background. In the reverse situation, we observed a trend toward increased Nrx-1 when overexpressing Syd-1 in the Syd-1 mutant background (Supplementary Fig. 6). Thus, it appears likely that complex formation with Syd-1 not only retains but also stabilizes Nrx-1.

Collectively, these data suggest that, as expected, Syd-1 promotes the anchoring of Liprin- $\alpha$ clusters. Syd- 1 also actively recruits and keeps Nrx-1 levels high in active zone-proximal compartments.

\section{Evidence for a spatially retrograde function of NIg1}

In vivo $\mathrm{NMJ}$ synapse assembly is a protracted process of roughly 10 hours at $25^{\circ} \mathrm{C}$ and finally reaches a mature steady state. Liprin- $\alpha$ and Syd-1 mark sites of synapse formation first. Postsynaptic GluRIIA follows next and finally BRP is incorporated ${ }^{5}$. To readdress this early assembly process, we imaged Syd- 1 and Liprin- $\alpha$ at 30 -min intervals in living Drosophila larvae (Fig. 6a). As expected ${ }^{5}$, Liprin- $\alpha$ and Syd-1 were tightly clustered (Fig. 6b). We noticed, however, that such Liprin- $\alpha-$ Syd-1 clusters often dissolved again (Fig. 6c). In contrast, such disassembly events were hardly ever observed for GluRIIA (ref. 6; data not shown). Apparently not all early Liprin- $\alpha-$ Syd- 1 clusters entered the irreversible assembly phase marked by GluRIIA accumulation.

To address the influence of postsynaptic Nlg1 on presynaptic assembly, we imaged Liprin- $\alpha-$ Syd-1 clusters in Nlg1 mutants (Fig. 6d). Notably, the distribution of Syd-1 (Supplementary Fig. 7) and Liprin- $\alpha$ (data not shown) seemed to rapidly change over time at individual Nlg1 mutant active zones. Likewise, Liprin- $\alpha-S y d-1$ clusters appeared highly mobile (Fig. 6e,f). We scored all mobile clusters,

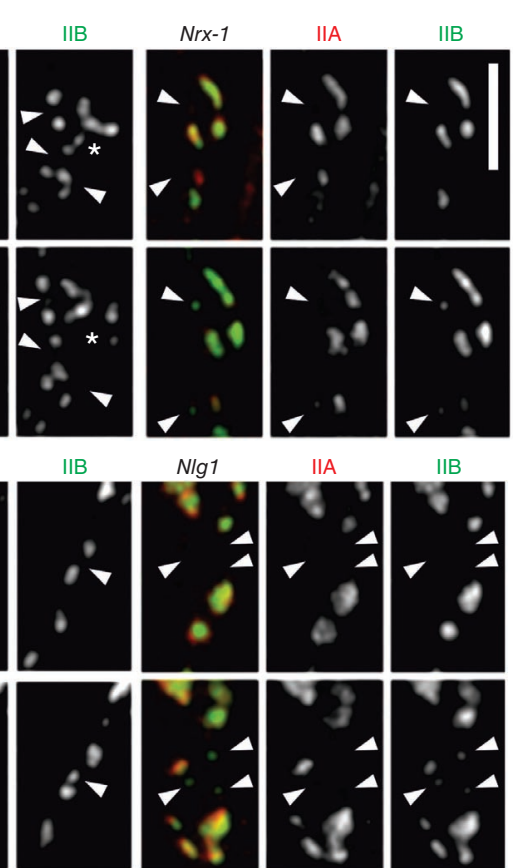

b IIB/IIA composition of new PSDs

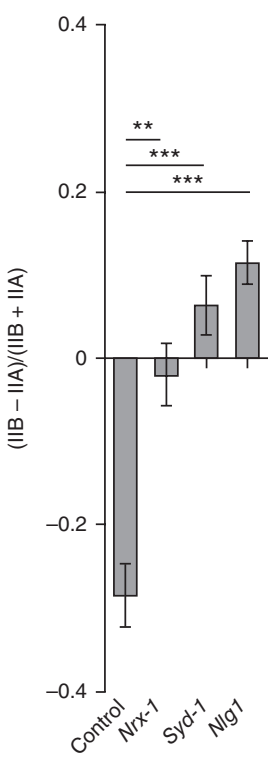

$\| \mathrm{A}$
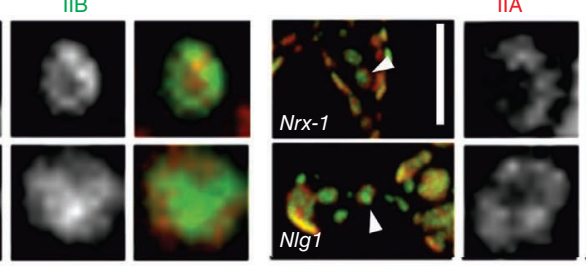

IIB
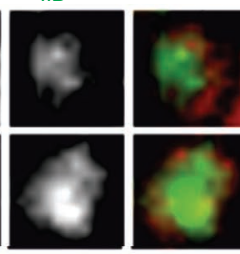

evaluating the image for each fluorophore (representing Syd-1 and Liprin- $\alpha$ ) separately as well as the two together. The overall mobility of each of these-Syd-1 spots, Liprin- $\alpha$ spots and Syd-1Liprin- $\alpha$ clusters - was significantly elevated in Nlg1 mutants over 60 min (Mann-Whitney $U$-tests: for Syd-1 spots, control: $0.079 \pm$ 0.006 mobile spots per $\mu \mathrm{m}$, mean \pm s.e.m., $n=8$; Nlg1: $0.176 \pm$ 0.013, $n=8$; control versus Nlg1 $P<0.001, U=0.0$; for Liprin- $\alpha$ spots, control: $0.080 \pm 0.007 n=8$; Nlg1: $0.189 \pm 0.021, n=8$; control versus Nlg1 $P<0.001, U=1.0$; for Liprin- $\alpha-S y d-1$ clusters, control: $0.052 \pm 0.009, n=8$; Nlg1: $0.155 \pm 0.017, n=8$, control versus Nlg1 $P<0.001, U=1.0$ ) Consistent with Nrx-1 serving as a hub for Nlg1 and Syd-1, we also found a tendency toward increased mobility of Liprin- $\alpha$ clusters in Nrx-1 mutants (Supplementary Fig. 5).

Together, these data suggest that Nlg1 is important for Syd-1Liprin- $\alpha$ cluster stabilization in a spatially retrograde fashion. Lack of postsynaptic Nlg1 seems to destabilize the corresponding presynaptic complex. Most likely this is due to deficits in the trans-synaptic promotion of Nrx-1 clustering in Nlg1 mutants (Fig. 3c,d), which ultimately leads to aberrant presynaptic active zones (Fig. 2).

GluR incorporation defects in Nrx-1, NIg1 and Syd-1 mutants Drosophila NMJ PSDs incorporate glutamate receptors containing either the GluRIIA or the GluRIIB subunit. Normally, most immature, young PSDs form by preferentially incorporating the GluRIIA complex, later followed by GluRIIB during subsequent PSD maturation ${ }^{6}$. Would presynaptic Nrx-1 and Syd-1 also influence postsynaptic assembly?

We addressed postsynaptic assembly at Syd-1, Nrx-1 and Nlg1 single mutant NMJs (Fig. 7a) by coexpressing fluorescently tagged 
GluRIIA and GluRIIB from genomic constructs ${ }^{6,29}$. Identified NMJs were reimaged after $24 \mathrm{~h}$ and PSDs were tracked over time in three dimensions. This method allowed us to identify newly formed PSDs. As expected, new PSDs in controls were predominantly rich in GluRIIA (Fig. 7a,b). By contrast, in Syd-1, Nrx-1 and Nlg1 single mutants, new PSDs were rich in GluRIIB (Fig. 7a,b). The early incorporation of GluRIIA, but not GluRIIB, is a rate-limiting driving force for PSD assembly and thus for synapse formation at growing NMJs $s^{6,29,30}$. This deficit in early GluRIIA incorporation might contribute to fewer synapses forming in these mutants (Fig. 1c,d).

GluRIIA incorporation appears to be nearly irreversible ${ }^{6}$. As a result, PSDs often show a GluRIIA-rich core surrounded by a GluRIIB-rich edge (Fig. 7c). Notably, this concentric arrangement was regularly inverted in all three-Nlg1, Nrx-1 and Syd-1-mutants (Fig. 7c), reflecting GluRIIB-rich PSDs that eventually incorporate GluRIIA (Supplementary Fig. 8a). As this suggests that a lack of $\mathrm{Nlg} 1$ is ultimately responsible for this inversion, we tested whether receptor organization in Nrx-1 mutants would profit from Nlg1 overexpression. Indeed, the receptor distribution largely normalized after Nlg1 overexpression (data not shown), indicating that lack of Nlg1 was in fact responsible for the PSD assembly deficits of $N r x-1$ mutants (and by extension also for those in Syd-1 mutants). Of note, a fraction of PSDs ultimately over-grew in all three mutants (Supplementary Fig. 8b,c).

In summary, Nlg1, Nrx-1 and Syd-1 mutants share a specific deficit in the early, PSD growth-promoting GluRIIA incorporation, and are likely directly responsible for this specific assembly deficit in the subcellular PSD distribution of GluR subunits. In our model, Syd-1 supports the aggregation of Nrx-1, which in turn clusters postsynaptic Nlg1, coupling pre- to postsynaptic assembly (Supplementary Fig. 9).

\section{DISCUSSION}

The Nrx and Nlg families include autism susceptibility genes ${ }^{31,32}$, and their proteins are needed for proper synapse formation during circuit development. It has so far, however, remained largely unclear how they molecularly integrate into the synapse formation process, particularly in regard to the assembly of the presynaptic active zone scaffold. Thus, identifying proteins coupling Nrx-Nlg to the assembly process itself and defining where in the sequence of events Nrx-Nlg acts is critical for a deeper understanding of synapse formation and remodeling.

Independent work in model organisms has identified and characterized proteins guiding active zone assembly, with Syd-1 proteins functioning upstream of Syd-2 (Liprin- $\alpha)^{5,15,16}$. In vivo imaging demonstrated that both Syd-1 and Liprin- $\alpha$ accumulate very early during synapse assembly (Supplementary Fig. 9) - earlier than postsynaptic GluRs, and much earlier than presynaptic $\mathrm{BRP}^{5}$. In vivo FRAP analysis now suggests that Syd-1 increases the dwell time of Nrx-1 near active zones and can actively recruit Nrx-1 in a PDZ-dependent manner (Supplementary Fig. 9). Likewise, Liprin- $\alpha$ cluster mobility was elevated in the Syd-1 mutant background, implying a retention function of Syd-1 for both Nrx-1 and Liprin- $\alpha$ at assembling active zones. This study also suggests that the assembly of initially forming Syd-1 and Liprin- $\alpha$ scaffolds is reversible (Supplementary Fig. 9). The success rate of establishing stable Syd-1 and Liprin- $\alpha$ scaffolds dropped in the absence of $\mathrm{Nlg} 1$ (Fig. 6d-f). As postsynaptic overexpression of Nlg1 increased the expression of presynaptic Nrx-1 (Supplementary Fig. 1a), interaction of these initial active zone scaffolds is likely to be directly dependent on local Nrx-1 interacting with Syd-1. It is tempting to speculate that the Nrx-1-Syd-1 interaction provides binding sites at newly forming active zones to drive the accumulation of Liprin- $\alpha$ scaffolds ${ }^{33,34}$ past a critical point, to enter an essentially irreversible maturation process (characterized by the onset of GluRIIA incorporation). Such a cooperative scheme might be optimized for the integration of regulatory elements and protect the system from untimely and aberrant assembly. In fact, the active zone component BRP has been shown to be under constitutive phosphorylation to avoid premature assembly $\mathrm{y}^{35,36}$.

We found here that mutants for Nlg1 and Nrx-1 showed aberrant active zone organization reflected in over-grown (star-shaped) T-bars (Fig. 2). We previously observed these phenotypes in Syd-1 mutants ${ }^{5}$. All three mutants (Syd-1,Nrx-1 and Nlg1) assemble fewer active zones per $\mathrm{NMJ}^{5,11,12}$. Consequently, levels of unused active zone scaffold components, such as BRP, might locally accumulate along their NMJ terminals. This increase in building blocks in turn might result in over-growth of the remaining active zone scaffolds. Additionally, Syd-1, Nrx-1 and Nlg1 might define an assembly sequence, which in turn could be a precondition to properly terminating assembly. One might speculate that an improperly assembled scaffold could retain free valences and that the scaffold could outgrow improperly. As active zone localization of Syd-1 clustering did not strongly depend on either Nrx-1 or Nlg1, we suspect that a complex of Syd-1 with Nrx-1 might be important for the regulation of BRP incorporation. Potentially, binding to Nrx-1 (in a trans-synaptic complex with Nlg1) might unmask additional domains of Syd-1 for assembly and thereby allow the effective stabilization of Liprin- $\alpha$ scaffolds. Notably, mammalian Nlg1 has also been implicated in induction and maturation of the presynaptic terminal ${ }^{37,38}$.

Nrx-1 (ref. 11) and Syd-1 (ref. 5) are both expressed throughout the CNS, whereas Nlg1 is not ${ }^{12}$. It is likely that other Drosophila Nlgs (see ref. 12) substitute for Nlg1 at central synapses. Of note, we found star-shaped T-bars at adult CNS synapses of Syd-1 mutants as well (data not shown), suggesting that similar mechanisms as here described for NMJ synapses apply to CNS synapses.

Although Syd-1 remains cytoplasmic and depends on the presence of Nrx-1 to localize to the plasma membrane in non-neural cells (salivary gland epithelial cells; Fig. $\mathbf{4 b}$-f), Syd-1 can also localize to active zones in the absence of Nrx-1. Consistently, Syd-1 mutated in its PDZ-domain (Gal4-UAS expressed) still localized to active zones, at least to a fair extent. Thus, nascent active zones seemingly contain additional proteins providing binding sites for Syd-1 (that also may be needed to stabilize a complex of Syd-1 and Nrx-1). Binding sites are still present after deletion of either Liprin- $\alpha$ or BRP-despite a direct interaction of Syd-1 with BRP . Additional proteins representing potential upstream functions, such as the adaptor protein Neurabin that was shown to recruit C. elegans Syd-1 and Syd-2 to F-actin foci ${ }^{39}$, are prime candidates for the localization of Syd-1.

Unlike those of endogenous Syd-1, levels of Gal4-UAS-expressed Syd-1 depended on the presence of Nrx-1 (Supplementary Fig. 6). Thus, uncomplexed, excessive Syd-1 might be subjected to degradation. Of note, Liprin- $\alpha$ is a downstream effector and possible substrate of the E3 ubiquitin ligase APC/C (ref. 40).

We found that early and rapid GluRIIA-mediated growth of nascent PSDs (younger than $24 \mathrm{~h}$ ) was selectively impaired in Syd-1, Nrx-1 and Nlg1 mutants, where young PSDs are characterized by a high GluRIIB content. Mutants for GluRIIA, but not for GluRIIB, fail to grow sufficient synapses per terminal when challenged by high-temperature rearing ${ }^{41}$. In addition, terminals of Syd-1, Nrx-1 and Nlg1 all suffer from under-growth of synaptic terminals ${ }^{5,11,12}$. Thus, this undergrowth might partially be a consequence of reduced initial GluRIIA incorporation. However, this leaves the question of how Nlg1 dictates GluRIIA incorporation. Nlg1 clusters, functionally associated with proteins regulating initial synapse assembly, might selectively promote 
GluRIIA incorporation directly (Supplementary Fig. 9). Notably, Nrx-Nlg complexes have been associated with GluR subunit-specific recruitment into PSDs in mammals. In that system, overexpression of Nlg1 selectively decreases the surface mobility of GluA2-containing AMPA-type glutamate receptors, in a manner mediated by a PSD95Nlg1 interaction, while having no effect on GluA1 homomers ${ }^{42,43}$. Indeed, Nlg1 is able to recruit the PSD95 ortholog Discs large (Dlg) to the Drosophila NMJ ${ }^{12}$. Nlg1 clustering instructed by Syd-1 and Nrx-1 might create a seed for GluRIIA clustering mediated by Dlg and other scaffold proteins. It should be noted, however, that GluRIIA receptors still incorporate at Nlg1-mutant PSDs, although at a later time point of assembly, with PSDs also overshooting in size (Supplementary Fig. 8c). Thus, Nlg1 seems particularly important for providing binding sites for GluRIIA complexes during early assembly, and choosing the right temporal sequence also seems important for the proper termination of the assembly process.

\section{METHODS}

Methods and any associated references are available in the online version of the paper.

Note: Supplementary information is available in the online version of the paper.

ACKNOWLEDGMENTS

We would like to thank C. Quentin and A. Stawrakakis for excellent technical assistance and R. Jahn for the use of equipment. T. Andlauer and U. Thomas critically read the manuscript. We further thank M. Bhat (University of North Carolina) and W. Xie (Southeast University, Nanjing) for generously sharing reagents. This work was supported by grants from the Deutsche Forschungsgemeinschaft to S.J.S. (Exc 257, SI849/2-1 and 2-2, TP A16/SFB 551, TP B23/SFB581, TP A3 and A6/SFB 958) and H.A. (AB 116/3-2). M.H. is funded by a European Research Council Starting Grant (Astrofunc).

\section{AUTHOR CONTRIBUTIONS}

D.O., O.K. and S.J.S. designed research. D.O., O.K., V.K.G., D.B., H.D., W.F., C.W., S.M. and E.R. performed experiments. D.O., O.K., V.K.G., D.B., H.D., W.F., C.W., S.M., H.A. and S.J.S. analyzed data. M.H. and S.E. shared protocols, reagents and advice. All authors commented on the paper. D.O., O.K. and S.J.S. wrote the paper.

\section{COMPETING FINANCIAL INTERESTS}

The authors declare no competing financial interests.

1. Owald, D. \& Sigrist, S.J. Assembling the presynaptic active zone. Curr. Opin. Neurobiol. 19, 311-318 (2009).

2. Fejtova, A. \& Gundelfinger, E.D. Molecular organization and assembly of the presynaptic active zone of neurotransmitter release. Results Probl. Cell Differ. 43, 49-68 (2006)

3. Jin, Y. \& Garner, C.C. Molecular mechanisms of presynaptic differentiation. Annu. Rev. Cell Dev. Biol. 24, 237-262 (2008).

4. Collins, C.A. \& DiAntonio, A. Synaptic development: insights from Drosophila. Curr. Opin. Neurobiol. 17, 35-42 (2007).

5. Owald, D. et al. A Syd-1 homologue regulates pre- and postsynaptic maturation in Drosophila. J. Cell Biol. 188, 565-579 (2010).

6. Schmid, A. et al. Activity-dependent site-specific changes of glutamate receptor composition in vivo. Nat. Neurosci. 11, 659-666 (2008)

7. Scheiffele, P., Fan, J., Choih, J., Fetter, R. \& Serafini, T. Neuroligin expressed in nonneuronal cells triggers presynaptic development in contacting axons. Cell 101, 657-669 (2000).

8. Graf, E.R., Zhang, X., Jin, S.X., Linhoff, M.W. \& Craig, A.M. Neurexins induce differentiation of GABA and glutamate postsynaptic specializations via neuroligins. Cell 119, 1013-1026 (2004)

9. Südhof, T.C. Neuroligins and neurexins link synaptic function to cognitive disease. Nature 455, 903-911 (2008).

10. Varoqueaux, F. et al. Neuroligins determine synapse maturation and function. Neuron 51, 741-754 (2006).
11. Li, J., Ashley, J., Budnik, V. \& Bhat, M.A. Crucial role of Drosophila neurexin in proper active zone apposition to postsynaptic densities, synaptic growth, and synaptic transmission. Neuron 55, 741-755 (2007).

12. Banovic, D. et al. Drosophila neuroligin 1 promotes growth and postsynaptic differentiation at glutamatergic neuromuscular junctions. Neuron 66, 724-738 (2010).

13. Sun, M. et al. Neuroligin 2 is required for synapse development and function at the Drosophila neuromuscular junction. J. Neurosci. 31, 687-699 (2011).

14. Fairless, R. et al. Polarized targeting of neurexins to synapses is regulated by their C-terminal sequences. J. Neurosci. 28, 12969-12981 (2008).

15. Dai, Y. et al. SYD-2 Liprin-alpha organizes presynaptic active zone formation through ELKS. Nat. Neurosci. 9, 1479-1487 (2006).

16. Patel, M.R. et al. Hierarchical assembly of presynaptic components in defined C. elegans synapses. Nat. Neurosci. 9, 1488-1498 (2006).

17. Patel, M.R. \& Shen, K. RSY-1 is a local inhibitor of presynaptic assembly in C. elegans. Science 323, 1500-1503 (2009).

18. Kaufmann, N., DeProto, J., Ranjan, R., Wan, H. \& Van Vactor, D. Drosophila liprinalpha and the receptor phosphatase Dlar control synapse morphogenesis. Neuron $\mathbf{3 4}$ 27-38 (2002).

19. Hell, S.W. Far-field optical nanoscopy. Science 316, 1153-1158 (2007).

20. Fouquet, W. et al. Maturation of active zone assembly by Drosophila Bruchpilot. J. Cell Biol. 186, 129-145 (2009).

21. Marrus, S.B. \& DiAntonio, A. Preferential localization of glutamate receptors opposite sites of high presynaptic release. Curr. Biol. 14, 924-931 (2004).

22. Kittel, R.J. et al. Bruchpilot promotes active zone assembly, Ca2+ channel clustering, and vesicle release. Science 312, 1051-1054 (2006).

23. Hallermann, S. et al. Naked dense bodies provoke depression. J. Neurosci. 30, 14340-14345 (2010).

24. Sun, M. et al. Genetic interaction between Neurexin and CAKI/CMG is important for synaptic function in Drosophila neuromuscular junction. Neurosci. Res. 64 362-371 (2009)

25. Lenfant, N. et al. A genome-wide study of PDZ-domain interactions in C. elegans reveals a high frequency of non-canonical binding. BMC Genomics 11, 671 (2010).

26. Swan, L.E. et al. Complex interaction of Drosophila GRIP PDZ domains and Echinoid during muscle morphogenesis. EMBO J. 25, 3640-3651 (2006).

27. Miller, K.E. et al. Direct observation demonstrates that Liprin-alpha is required for trafficking of synaptic vesicles. Curr. Biol. 15, 684-689 (2005).

28. Spangler, S.A. \& Hoogenraad, C.C. Liprin-alpha proteins: scaffold molecules for synapse maturation. Biochem. Soc. Trans. 35, 1278-1282 (2007).

29. Rasse, T.M. et al. Glutamate receptor dynamics organizing synapse formation in vivo. Nat. Neurosci. 8, 898-905 (2005).

30. Sigrist, S.J., Thiel, P.R., Reiff, D.F. \& Schuster, C.M. The postsynaptic glutamate receptor subunit DGluR-IIA mediates long-term plasticity in Drosophila. J. Neurosci. 22, $7362-7372$ (2002)

31. Lisé, M.F. \& El-Husseini, A. The neuroligin and neurexin families: from structure to function at the synapse. Cell. Mol. Life Sci. 63, 1833-1849 (2006).

32. Jamain, S. et al. Reduced social interaction and ultrasonic communication in a mouse model of monogenic heritable autism. Proc. Natl. Acad. Sci. USA 105, 1710-1715 (2008).

33. Taru, H. \& Jin, Y. The Liprin homology domain is essential for the homomeric interaction of SYD-2/Liprin-alpha protein in presynaptic assembly. J. Neurosci. 31 16261-16268 (2011)

34. Wei, Z. et al. Liprin-mediated large signaling complex organization revealed by the liprin-alpha/CASK and liprin-alpha/liprin-beta complex structures. Mol. Cell $\mathbf{4 3}$ 586-598 (2011).

35. Nieratschker, V. et al. Bruchpilot in ribbon-like axonal agglomerates, behavioral defects, and early death in SRPK79D kinase mutants of Drosophila. PLoS Genet. 5 e1000700 (2009).

36. Johnson, E.L. III, Fetter, R.D. \& Davis, G.W. Negative regulation of active zone assembly by a newly identified SR protein kinase. PLOS Biol. 7, e1000193 (2009).

37. Futai, K. et al. Retrograde modulation of presynaptic release probability through signaling mediated by PSD-95-neuroligin. Nat. Neurosci. 10, 186-195 (2007).

38. Wittenmayer, N. et al. Postsynaptic Neuroligin1 regulates presynaptic maturation. Proc. Natl. Acad. Sci. USA 106, 13564-13569 (2009).

39. Chia, P.H., Patel, M.R. \& Shen, K. NAB-1 instructs synapse assembly by linking adhesion molecules and F-actin to active zone proteins. Nat. Neurosci. 15, 234-242 (2012).

40. van Roessel, P., Elliott, D.A., Robinson, I.M., Prokop, A. \& Brand, A.H. Independent regulation of synaptic size and activity by the anaphase-promoting complex. Cel/ 119 , 707-718 (2004)

41. Schmid, A. et al. Non-NMDA-type glutamate receptors are essential for maturation but not for initial assembly of synapses at Drosophila neuromuscular junctions. J. Neurosci. 26, 11267-11277 (2006).

42. Mondin, M. et al. Neurexin-neuroligin adhesions capture surface-diffusing AMPA receptors through PSD-95 scaffolds. J. Neurosci. 31, 13500-13515 (2011).

43. Heine, M. et al. Activity-independent and subunit-specific recruitment of functional AMPA receptors at neurexin/neuroligin contacts. Proc. Natl. Acad. Sci. USA 105 20947-20952 (2008). 


\section{ONLINE METHODS}

Genetics and molecular cloning. Fly strains were reared under standard laboratory conditions. For all experiments, both male and female larva were used for analysis. All mutations were kept in the $w 1118$ genetic background. Double mutant combinations were verified via genomic $\mathrm{PCR}^{5,11,12}$ or, where possible, by complementation analysis. Mutants for Syd-1 (Syd-1 ex1.2/Syd-1 ex3.4 $)^{5}, \mathrm{Nrx}-1$ $\left(N r x-1^{241} / N r x-1 D f\right)^{11}, N l g 1\left(N \lg 1^{\text {ex1.9}} / N \operatorname{Ng} 1^{\text {ex2.3 }} \text { or Nlg1 } 1^{1960} / N_{l g} 1^{H 324}\right)^{12}$, Liprin- $\alpha$

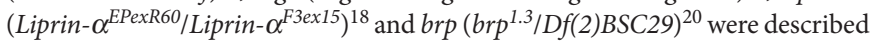
previously. Flies carrying UAS-GFP-tagged Nrx-1 (ref. 12), UAS-untagged-Nrx-1 (ref. 24), UAS-untagged-Nrx-1 ${ }^{\mathrm{PDB}} *$ (ref. 24), UAS-GFP or -mStraw-tagged Syd-1 (ref. 5), UAS-GFP-tagged Liprin- $\alpha$ (ref. 20), UAS-GFP-tagged-Nlg1 (ref. 12) and UAS-GFP-tagged-Nlg1 $\Delta$ Cyto (ref. 12) were described previously. mStrawberrytagged UAS-Liprin- $\alpha$ was obtained using the Gateway vector pTWmStrawberry and $p E N T R 4 /$ Liprin- $\alpha$ (ref. 20). The D358R point mutation was introduced to GFP-tagged-Nlg1 $\Delta$ Cyto as previously described ${ }^{12}$. UAS-Syd- $1^{\text {PDZ }}{ }^{*}$ was constructed based on the UAS-Syd-1cDNA (also referred to as Syd-1 Rescue) ${ }^{5}$. Two fragments were obtained via PCR using (1) $5^{\prime}$-GAGCGCGGCCGCGATGACG$3^{\prime}$ and $5^{\prime}$-AGCCCGCCGTCTGGCCCGGCGCCTTGACTA- $3^{\prime}$, and (2) $5^{\prime}$-TA GTCAAGGCGCCGGGCCAGACGGCGGGCTTGT- $3^{\prime}$ and $5^{\prime}$-CCGTTGAC ATTCTTCTCGAGGGACCCA-3' ${ }^{\prime}$, and joined using elongation PCR to create the $\mathrm{PDZ}^{*}$ construct. UAS-Syd-1cDNA and -PDZ* were digested using NotI and XhoI and ligated. $p$ TmStrawberryW/Syd-1 $1^{P D Z^{*}}$ was obtained using the Gateway system (Invitrogen). Point mutations were confirmed via double strand sequencing. For the yeast two-hybrid bait vector, a region encoding the cytoplasmic $\mathrm{C}$ terminus of Nrx-1 was obtained via PCR on UAS-Nrx-1-cDNA (ref. 12) using 5'-GATGCCATGGAGTCGAATGGCGATCGTGGCT-3' and $5^{\prime}$-GTCTATGAA TTCGTTTACACATACCACTCCTTGACGTCCT- $3^{\prime}$. The product was digested using NcoI and EcoRI and ligated into pGADT7-IIB (modified $p G A D T 7$, containing only a single NcoI site). For the yeast two-hybrid prey vectors, a region encoding the Syd-1 PDZ domain was obtained via PCR on UAS-Syd-1cDNA and UAS-Syd-1 ${ }^{\text {PDZ }}{ }^{*} D N A$, respectively, using 5'-GTCTATGAATTCCTGGTAGAAA

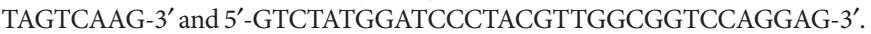
The products were digested using EcoRI and BamHI and ligated into $p G B K T 7$.

Immunostainings and imaging. The relative number of synaptic boutons was quantified on dorsal muscles 1 and 9 in abdominal segment A3 of intact CD8GFP-Sh third-instar larvae expressing UAS-Nlg1 $\Delta$ Cyto GFP (Mef2-Gal4) in various genetic backgrounds. The relative number of synaptic boutons was calculated by adjusting the absolute bouton number to the approximate muscle surface areas.

Larval filets were dissected and stained as described previously ${ }^{5}$. The following primary antibodies were used: Rb $\alpha$ DSyd-1 (ref. 5; 1:500), Rb $\alpha$ GluRIID (ref. 5; 1:500), M $\alpha \mathrm{Nc} 82$ (refs. 20,22; 1:100) (provided by E. Buchner, Universität Würzburg), RboNlg1 (ref. 12; 1:500) and Gp $\alpha$ Nrx-1 (ref. 11; 1:500) (provided by M. Bhat, School of Medicine, University of North Carolina). Secondary antibodies

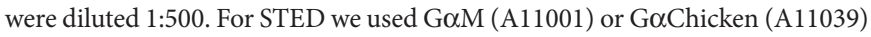
Alexa488 (Molecular Probes, Germany).

Nlg1 and Nrx-1 clusters were quantified from confocal stacks of NMJs converted to maximal projections. The signal of a Cy5 $\alpha$ HRP antibody (23-175-021, Jackson ImmunoResearch, 1:250) was used as template for a mask, restricting the quantified area to the shape of the NMJ. After subtraction of background signals (where indicated), the absolute intensity per unit $\mathrm{NMJ}$ area was acquired using Image J and converted to the relative intensity in Microsoft Excel.

In vivo imaging: All UAS constructs (except for Nlg1 constructs (Mef2-Gal4, see above)) were driven in motor neurons using ok6-Gal4 ( $\{$ GawB $\} O K 6$; ref. 44). NMJs on muscles 26 and 27 were recorded. Salivary glands were also imaged from living larvae. All live imaging was performed as previously described ${ }^{5,6,20}$. Briefly, larvae of different genotypes were mounted in the imaging chamber and single confocal sections were acquired. For FRAP experiments (all performed in the synaptic area of the NMJ), intense laser light was applied to a region of interest, bleaching green or/and red fluorescent fusion tags. The after-photobleaching image was taken approximately $1-2 \mathrm{~min}$ after the $t=0 \mathrm{~min}$ image. After an incubation time of $30 \mathrm{~min}$, the junctions were reimaged and compared with the images taken before bleaching. For 30-min time-course experiments, larvae were awakened by applying fresh air but remained in the imaging chamber between the acquisition points. For 24 -h imaging intervals, the larvae were reared in an incubator at $25^{\circ} \mathrm{C}$ between acquisitions.

FRAP analysis was done following a previously described protocol ${ }^{20}$.
Quantification of mobile Liprin- $\alpha$ and Syd-1 spots was performed on a time series of three confocal stacks of individual junctions that were sequentially acquired at 30 - $\mathrm{min}$ intervals ( $t=0 \mathrm{~min}, t=30 \mathrm{~min}, t=60 \mathrm{~min}$ ). These images were deconvolved using Autoquant X 2.1.1 from MediaCybernetics. Confocal stacks were merged into a single plane by using the maximum projection function of ImageJ. Subsequently, a Gaussian blur filter was applied (blur radius, 0.5 pixels) and the highest signal intensity was scaled to 255 . Only spots with a mean intensity 2.5 times higher than the background intensity were counted. The spots were classified as mobile spots or immobile spots. Spots were considered to be 'immobile' spots if they remained at their initial position (relative to the surrounding spots) during all three time points, whereas 'mobile' spots were defined as spots that appeared and/or disappeared during the time interval of $60 \mathrm{~min}$. All spots were manually counted, and the number of spots was normalized to the length of the junction (estimated using the line function in ImageJ).

For quantitative analysis of GluR fields the following genotypes were produced: [Df $(2 \mathrm{~L})^{\mathrm{h} 4}$, GluRIIB-GFP/A22,GluRIIA-mRFP] for controls, $\left[\mathrm{Df}(2 \mathrm{~L})^{\mathrm{h} 4}\right.$,GluRIIB-GFP/A22, GluRIIA-mRFP; Syd-1 $1^{\text {ex1.2 }} /$ Syd-1 $\left.{ }^{\text {ex3.4 }}\right]$ for Syd-1, $\left[\operatorname{Df}(2 \mathrm{~L})^{\mathrm{h} 4}\right.$,GluRIIB-GFP/A22, GluRIIA-mRFP; Nrx-1 $\left.1^{241} / N r x-1^{241}\right]$ for $N r x-1$, $\&\left[\mathrm{Df}(2 \mathrm{~L})^{\mathrm{h} 4}\right.$, GluRIIB-GFP/A22,GluRIIA-mRFP; Nlg1 $\left.1^{\mathrm{ex} 2.3} / N \lg 1^{\mathrm{ex} 1.9}\right]$ for Nlg1 mutants.

The GluRIIA,GluRIIB (Df(2L) $\left.{ }^{\mathrm{h} 4} / \mathrm{A} 22\right)$ double mutation was rescued by the expression of fluorescently tagged genomic constructs of GluRIIA and GluRIIB under the control of their endogenous promoters ${ }^{6}$.

Confocal image stacks were analyzed using Image and Imaris 6.15. 3Dsurface masks were generated after background subtraction, scaling to the highest pixel intensity and application of Gaussian blur filters. These stacks were further analyzed in Imaris and single receptor fields (PSDs) were detected using the seed point detection algorithm combined with manual segmentation. Pixel intensities of unprocessed GluRIIA and GluRIIB channels in single PSDs were transferred to Microsoft Excel for further processing, as previously described ${ }^{6,12}$.

Receptor topology at individual PSDs and GluRIIA intensities in control, Nrx-1, Syd-1 and Nlg1 larvae were evaluated in images acquired from muscle 4 of fixed preparations and analyzed as described previously ${ }^{12}$.

All confocal images were acquired on a Leica TCS-SP5 microscope with a $63 \times$ magnification, NA 1.4 oil objective using Application Suite Advanced Fluorescence (LAS AF; Leica) software. Confocal stacks were processed with ImageJ software (http://rsbweb.nih.gov/ij/), Imaris 6.15 (Bitplane) and Autoquant X 2.1.1 (MediaCybernetics).

STED and electron microscopy. STED images were acquired on a Leica TCS STED CW. Images were deconvolved using the built-in deconvolution algorithms of the Leica LAS-AF software. The PSF was generated by using a 2D Lorentz function with the full-width half-maximum set to $80 \mathrm{~nm}$ (as calculated on the image using the Wiener filter algorithm; regulation parameter: 0.05 ). For preparation of the figures, a Gaussian blur filter of 0.5 was applied to the images.

Measurement of BRP ring diameters: deconvolved STED images of BRP stained NMJs (muscle 4) of $3^{\text {rd }}$ instar larvae were processed in ImageJ. The diameters of planar oriented BRP rings were measured using the line tool of ImageJ. The distance from intensity maximum to intensity maximum was acquired in the plot window of individual hand-drawn lines and transferred to Microsoft Excel. Per genotype, 5-7 images were analyzed, resulting in 36-56 measurements per group.

Electron microscopy: Conventional transmission electron microscopy and $3 \mathrm{D}$-serial reconstruction were conducted as described ${ }^{5}$.

Coimmunoprecipitation and yeast two-hybrid assay. For protein extracts used in coimmunoprecipitation experiments, we carried out a Drosophila head fractionation based on protocols from mammalian subcellular preparations ${ }^{45}$. In brief, in the absence of detergents, Drosophila wild-type heads were sheared mechanically and differential centrifugation was applied to separate particles according to their size and density. Recovered membranes were then solubilized in aqueous immunoprecipitation buffer containing $20 \mathrm{mM}$ HEPES pH 7.4, $200 \mathrm{mM}$ $\mathrm{KCl}, 2 \mathrm{mM} \mathrm{MgCl} 2,1 \%$ Triton X-100. Precleared protein extract was incubated for $10 \mathrm{~h}$ at $4{ }^{\circ} \mathrm{C}$ with either M $\alpha \mathrm{Nrx}-1$ (mAb4, provided by W. Xie, Southeast University, Nanjing, China ${ }^{24}$ ) or murine IgGs (Sigma-Aldrich) coupled to Protein A Sepharose-beads (Bio-Rad). After washing four times with immunoprecipitation buffer, proteins were eluted with $50 \mu \mathrm{l} 2 \times$ SDS-PAGE sample 
buffer. For immunoblot analysis, $4 \%$ of input and $10 \mu \mathrm{l}$ of the eluate were loaded. Membranes were probed with M $\alpha$ Nrx-1 (ref. 24) and Rb $\alpha$ Syd-1 (ref. 5).

For the yeast two-hybrid assay, Saccharomyces cerevisiae reporter strain Y187 (Clontech) was transformed with the Nrx-1 bait vector and prey vectors containing the unmutated PDZ domain or the mutated domain (PDZ*) of Syd-1. Clontech's control vectors (pGBKT7-Lam, pGBKT7-53 and pGADT7-T) were used as the negative and positive controls. Liquid cultures were assayed for $\beta$-galactosidase using ONPG ( $o$-nitrophenyl- $\beta$-D-galactopyranoside) to compare for relative strength of the protein-protein interaction. The assay was performed as described in the Yeast Protocols Handbook (Clontech) with the exceptions that cells were permeabilized with SDS/chloroform ${ }^{46}$ and that $\mathrm{OD}_{405}$ was measured. Clones showing $\beta$-galactosidase activity twice that of the negative control values were analyzed.
Statistics. Data were analyzed with Prism (GraphPad Software). To compare two groups, nonparametric Mann-Whitney $U$-tests were used for all data sets. For comparison of more than two groups, nonparametric Kruskal-Wallis tests were used, followed by a Dunn's multiple comparison test. $P$ values, $n$ values, and $U$ or $K$ statistic are given in the figure legends or main text.

44. Aberle, H. et al. Wishful thinking encodes a BMP type II receptor that regulates synaptic growth in Drosophila. Neuron 33, 545-558 (2002).

45. Huttner, W.B., Schiebler, W., Greengard, P. \& De Camilli, P. Synapsin I (protein I), a nerve terminal-specific phosphoprotein. III. Its association with synaptic vesicles studied in a highly purified synaptic vesicle preparation. J. Cell Biol. 96, 1374-1388 (1983).

46. Guarente, L. Yeast promoters and lacZ fusions designed to study expression of cloned genes in yeast. Methods Enzymol. 101, 181-191 (1983). 